\title{
Nuove prospettive di ricerca sulle tematiche di Management Control
}

\author{
Luciano Marchi*
}

In una fase di rapido cambiamento degli scenari ambientali e aziendali, è necessario riflettere se e come la ricerca nell'area dei sistemi di Management Control debba essere ri-orientata e, quindi, se la linea editoriale della rivista debba essere ri-definita.

Dare risposta alle richieste del mondo accademico e degli operatori aziendali sulle tematiche di pianificazione, informazione e controllo, con il ricorso ad un approccio integrato di analisi, era stata la linea editoriale definita nel primo numero della rivista (Management Control, n. 1/2011).

Il suddetto approccio di analisi era stato definito in termini di "integrazione dei sistemi operativi con quelli informativo-contabili, della struttura organizzativa con la tecnologia informatica, dell'analisi revisionale con quella prospettica, anche al fine di raccordare i sistemi di misurazione con quelli di governo aziendale, nel riferimento indistinto ad aziende pubbliche e private, profit e non-profit”.

La rivista intendeva differenziarsi, in tal modo, dalle riviste "generaliste” dell'economia aziendale e dall'insieme, più numeroso, delle riviste aventi carattere specialistico in rapporto ad una singola funzione aziendale, ovvero ad uno specifico settore di attività.

L'obiettivo di fondo della rivista era quello di inserirsi nel processo di internazionalizzazione della ricerca coniugando le conoscenze affermate a livello internazionale con i valori di fondo del pensiero economico aziendale italiano, anche nella scelta metodologica, cioè mantenendo il noto metodo di ricerca integrato induttivo-deduttivo.

Il richiamo all'approccio integrato di analisi e all'obiettivo di fondo ci permette di dare una prima risposta alla domanda che ci siamo posti all'inizio: la linea editoriale della rivista rimane senz'altro valida.

La prospettiva dell'internazionalizzazione, tuttavia, ha assunto un ruolo sempre più dominante, inflenzando i metodi ed i contenuti dei lavori sotto-

\footnotetext{
*Università di Pisa, Dipartimento di Economia e Management, luciano.marchi@unipi.it.
} 
posti alla rivista e determinando un progressivo distacco degli studi di $M a-$ nagement Control dalla nostra tradizione economico-aziendale.

In particolare, con riferimento alla metodologia, sempre più spesso si ricorre alla dimostrazione analitica delle ipotesi di ricerca, con l'approccio sperimentale tipico delle scienze "dure". Vengono proposte, dunque, ipotesi di ricerca sempre più "selettive", per verificare relazioni di causa-effetto nella logica vero-falso, con il ricorso alle basi di dati disponibili ovvero ad indagini campionarie, trascurando spesso l'accuratezza dei dati disponibili e di quelli raccolti su base campionaria. Anche il linguaggo utilizzato è sempre più quello matematico, poco adatto alla comunicazione nella comunità scientifica degli aziendalisti, perchè trascura le variabili qualitative, l'interpretazione dei dati e la comprensione delle relazioni di concausa ed effetto molteplice tra gli elementi considerati.

Relativamente ai contenuti, le tematiche rilevanti diventano non quelle legate alle comprensione della complessità aziendale, ma quelle che meglio si prestano ad analisi di correlazione o di "content analysis"; di fatto ci si focalizza, di nuovo, sulla metodologia considerata a priori migliore, determinando sempre più spesso un distacco dalla realtà aziendale.

In conclusione, considerando ancora valida la linea editoriale della rivista precedentemente definita e riaffermando l'orientamento di fondo (raccogliere la sfida dell'internazionalizzazione senza abbandonare la nostra tradizione economico-aziendale), riteniamo necessari alcuni interventi volti a garantire la fruibilità della rivista sia nella comunità scientifica degli aziendalisti che nella comunità economico-sociale, con particolare riferimento ai soggetti attivamente coinvolti nei sistemi di governo e controllo aziendale.

La scelta è dunque quella di intervenire non sulla linea editoriale, ma sulla sua applicazione, operando, tra gli altri, i seguenti assestamenti:

1) accogliere articoli "ad invito" su tematiche rilevanti per gli operatori aziendali, attivando la sezione "Best Practices". Meglio interpretare i comportamenti migliori andando "in profondita" su casi aziendali, infatti, piuttosto che dare veste formale a correlazioni ad alto impatto comunicativo ma di scarso valore applicativo;

2) ri-attivare la pratica delle note esplicative e di raffronto teorico commentato a piè di pagina, riducendo i riferimenti bibliografici inseriti (tra parentesi) all'interno del testo;

3) negli articoli che utilizzano metodi quantitativi, eliminare nel testo la dimostrazione analitica, spostandola in appendice. 
Tutto ciò premesso, due sono le parole chiave che legano gli articoli contenuti nel presente numero di Management Control: innovazione ed integrazione.

L'innovazione e l'integrazione devono caratterizzare i sistemi informativi e, più in generale, i sistemi di controllo interno e possono essere osservate da diversi punti di vista, ai vari livelli, nella prospettiva sia interna che esterna.

Nel primo capitolo, di Francesco Bellini e Fabrizio D'Ascenzio, l'innovazione è rappresentata dalla dematerializzazione in termini di fatturazione elettronica; l'integrazione è interpretata, invece, sulla supply chain e sull'intera financial value chain, attraverso un insieme di indicatori di performance in grado di "monitorare" le indicate catene del valore nelle diverse prospettive, finanziarie e strategiche, del fornitore, dell'ordine e della consegna.

Nel secondo capitolo, l'innovazione e l'integrazione sono considerate congiuntamente in termini di controllo relazionale. Partendo dalla descrizione dei meccanismi, formali e informali, che caratterizzano i sistemi di controllo manageriale, l'autore ha condotto una survey su un campione di 249 organizzazioni di Destination Management per verificare i meccanismi e gli strumenti effettivamente utilizzati per il controllo relazionale ovvero gli ostacoli (concettuali ed economici) che impediscono o differiscono la loro implementazione.

Nel terzo capitolo, la prospettiva innovativa del controllo relazionale è trattata a partire dalla reputazione, vista come asset strategico nella misurazione delle performance aziendali, interpretando anche il suo impatto sul comportamento degli stakeholders.

Nei quarto capitolo, Katia Corsi e Daniela Mancini affrontano il tema della regulatory compliance dei sistemi di controllo interno e le sue implicazioni sui comportamenti degli operatori di area contabile. Più in particolare, viene indagato il ruolo dei "contabili" e le competenze che essi impiegano nell'adozione delle leggi e della regolamentazione. L'idea di base è che la compliance sia un'opportunità strategica, capace di attivare cambiamenti significativi nelle procedure contabili, negli strumenti informativi, nei ruoli organizzativi e nei meccanismi di controllo.

Il quinto capitolo, sulle cause e sulle implicazioni dei cambiamenti dell'auditor, fa riferimento alla situazione degli Stati Uniti d'America dove i cambiamenti sono volontari ed è prevista una comunicazione obbligatoria sui motivi del cambiamento. I risultati dell'analisi indicano che i cambiamenti permettono ai revisori di organizzare meglio il lavoro di audit, realiz- 
zando un bilanciamento tra le diverse aspettative del management e degli stakeholders.

Nell'ultimo capitolo, nella sezione delle Best Practices, Alessandro Musso analizza le problematiche relative al raccordo tra la contabilità manageriale e la contabilità dei costi, nell'ambito del processo di budgeting che ne esalta caratteristiche e logiche: la prima utilizza regole, procedure e schemi di rendiconto per identificare, a livello di business, i risultati reddituali che interessano ai manager operativi; la seconda è finalizzata a calcolare il costo di prodotto/servizio. Affrontando il problema a partire dalla distinta base di produzione, Musso si chiede, in particolare, quanto tali differenze siano accettabili e perché.

L'articolo di Musso, affrontando il problema in termini di prassi delle aziende, pone alcune interessanti domande e fornisce prime risposte al problema del necessario raccordo tra le diverse componenti dei sistemi contabili nell'ottica gestionale. L'auspicio è che, sul tema, si possa sviluppare un proficuo dibattito tra gli studiosi di Management Control. 